\title{
ORGANIZAÇÕES E PESSOAS PARTICIPANTES DO CICLO DE PREMIAC̣ÃO 1998
}

Encerrado o período de inscrições para o Ciclo de Premiação 1998 do Programa GESTÃO PÚBLICA E CIDADANIA, 631 programas, projetos ou atividades haviam enviado material e estavam aptos a participar do processo seletivo para a fase subseqüente.

A partir daí, a análise do material mobilizou um Comitê Técnico formado por um conjunto de militantes de organizações da sociedade civil e de pesquisadores de várias universidades e de outras instituições de pesquisa, todos eles de alguma forma atuantes na busca da melhoria da administração pública brasileira. Subdividido em grupos de quatro ou cinco pessoas, este Comitê organizou-se de forma a garantir que cada iniciativa governamental inscrita fosse analisada e discutida, preliminarmente, por dois grupos diferentes. A partir daí, uma nova rodada de discussão geral, na qual cada caso foi exaustivamente debatido, permitiu que se chegasse à indicação dos semifinalistas com base na construção gradual de consensos.

Uma vez indicados os 100 semifinalistas, foi-lhes solicitada a resposta a um questionário-padrão composto de 17 perguntas (ver quadro III, p.24). Estas respostas subsidiaram a análise para indicação dos 20 finalistas em uma nova bateria de discussões, em processo idêntico ao da etapa anterior. Escolhidas estas 20 iniciativas, um conjunto de especialistas e estudiosos foi a campo conhecer as experiências in loco, preparando então "Relatórios de Visita de Campo". Todo o material recolhido e produzido ao longo deste processo passou a compor o Banco de Dados do Programa, estando disponível a qualquer interessado para consulta e obtenção de cópias.

O Ciclo de Premiação de 1998 encerrou-se com um evento realizado na Fundação Getulio Vargas, em São Paulo, no qual os responsáveis pelas 20 iniciativas finalistas apresentaram ao público presente e debateram com uma Banca Julgadora, os programas desenvolvidos pelas organizações públicas nas quais atuam. Esta Banca reuniu-se ao final do dia e indicou cinco destaques, premiados com R\$10 mil, cabendo aos outros 15 finalistas prêmios de $R \$ 3$ mil.

A seguir, apresentamos as organizações e pessoas que de forma mais ativa estiveram envolvidas em cada uma das etapas deste esforço coletivo para identificar e disseminar os caminhos que vêm sendo percorridos para melhorar a administração pública brasileira. 


\section{COORDENAÇÃO DO PROGRAMA}

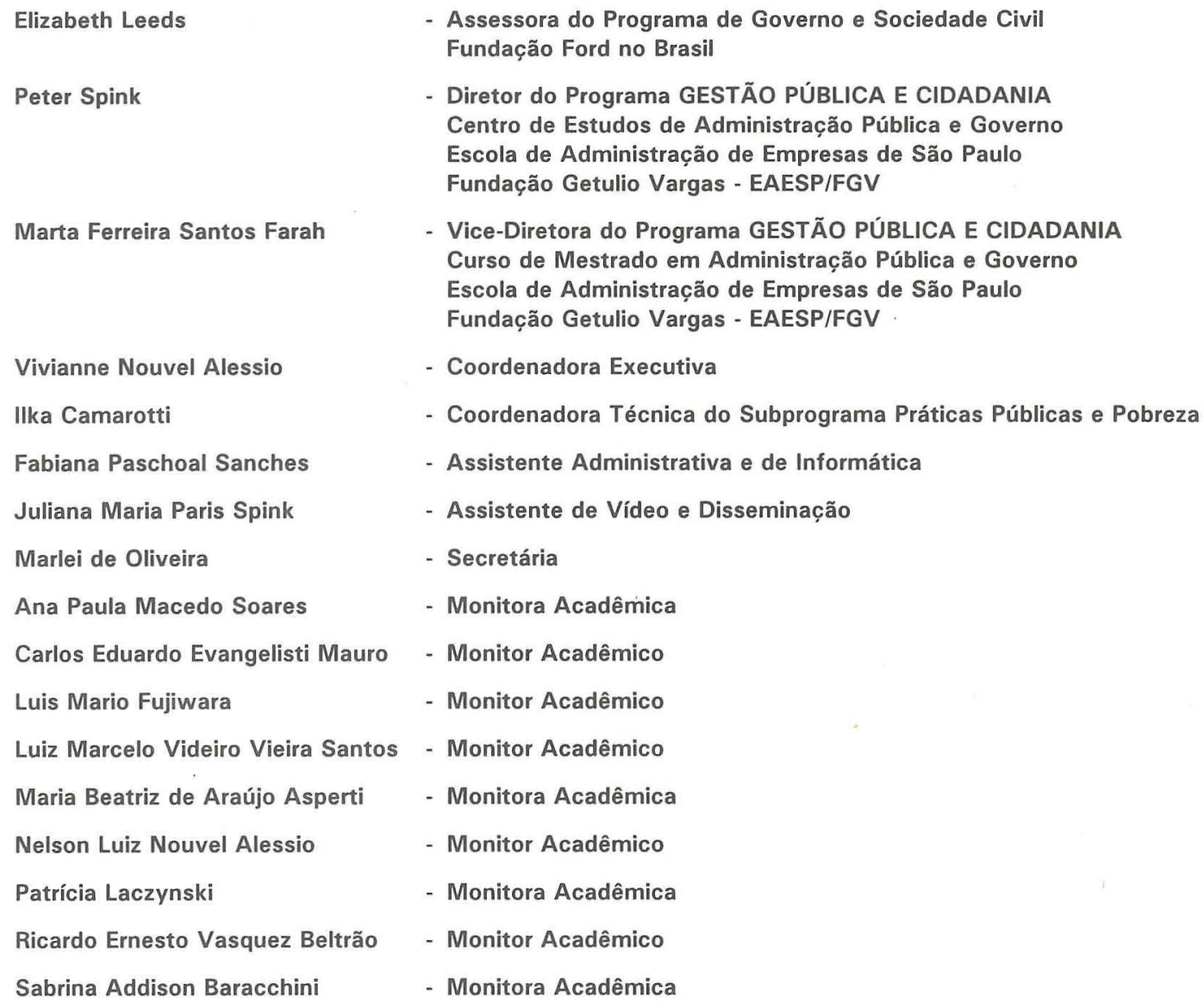

- Coordenadora Executiva

Ilka Camarotti

- Coordenadora Técnica do Subprograma Práticas Públicas e Pobreza

Fabiana Paschoal Sanches

- Assistente Administrativa e de Informática

Juliana Maria Paris Spink

- Assistente de Vídeo e Disseminação

Marlei de Oliveira

- Secretária

Ana Paula Macedo Soares

- Monitora Acadêmica

Carlos Eduardo Evangelisti Mauro

Luis Mario Fujiwara

- Monitor Acadêmico

Luiz Marcelo Videiro Vieira Santos

Maria Beatriz de Araújo Asperti

- Monitor Acadêmico

Nelson Luiz Nouvel Alessio

- Monitor Acadêmico

Patrícia Laczynski

- Monitora Acadêmica

- Monitor Acadêmico

Ricardo Ernesto Vasquez Beltrão

- Monitora Acadêmica

- Monitor Acadêmico

Sabrina Addison Baracchini

- Monitora Acadêmica

BANCA JULGADORA

Beatriz Azeredo

Carlos Alberto D' Oliveira

Claudett de Jesus Ribeiro

Elizabeth Varga

John Garrison

Juliana Junqueira

Plínio de Assis Pereira Júnior

Silvio Kaloustian
- Diretora do Banco Nacional de Desenvolvimento Econômico e Social - BNDES

- Diretor do Instituto Brasileiro de Administração Municipal - IBAM

- Representante dos 20 projetos finalistas de 1997 Projeto Auto-Estima das Crianças Negras, da Fundação da Criança e do Adolescente - FUNAC, do Estado do Maranhão

- Diretora do IDELI - Instituto de Desenvolvimento de Limeira

- Especialista em Análise Social e Sociedade Civil do Banco Mundial

- Jornalista de O Estado de São Paulo

- Coordenador do Departamento de Estudos e Atuação Social do Centro de Estudos Religiosos e Investigação Social - CERIS

- Oficial de Projetos da Organização das Nações Unidas para a Infância - UNICEF 
COMITÊ TÉCNICO - 1a FASE (INDICAÇÃO DOS SEMIFINALISTAS)

\begin{tabular}{|c|c|}
\hline Ailton Krenak & - Núcleo de Cultura Indígena \\
\hline Ana Maria Malik & $\begin{array}{l}\text { - Escola de Administração de Empresas de São Paulo - } \\
\text { Fundação Getulio Vargas - EAESP/FGV }\end{array}$ \\
\hline David Felipe Hastingt & $\begin{array}{l}\text { - Escola de Administração de Empresas de São Paulo - } \\
\text { Fundação Getulio Vargas - EAESP/FGV }\end{array}$ \\
\hline Edna M. Ramos de Castro & - Núcleo de Altos Estudos Amazônicos - Universidade Federal do Pará \\
\hline Fernando Guilherme Tenório & $\begin{array}{l}\text { - Escola Brasileira de Administração Pública - } \\
\text { Fundação Getulio Vargas - EBAP/FGV }\end{array}$ \\
\hline Heitor Paulo Battaglia & - Mestre em Administração Pública e Governo - EAESP/FGV \\
\hline Illka Camarotti & - Subprograma Práticas Públicas e Pobreza - EAESP/FGV \\
\hline Jorge Caiano & - PóLIS - Instituto de Estudos, Formação e Assessoria em Políticas Sociais \\
\hline José Antônio Gomes de Pinho & $\begin{array}{l}\text { - Núcleo de Pesquisa e Pós-Graduação em Administração - } \\
\text { Universidade Federal da Bahia }\end{array}$ \\
\hline Laura da Veiga & - Escola de Governo de Minas Gerais - Fundação João Pinheiro \\
\hline Luís Carlos Merege & - Centro de Estudos do Terceiro Setor - CETS - EAESP/FGV \\
\hline Luis Mario Fujiwara & - Mestrando em Administração Pública e Governo - EAESP/FGV \\
\hline Luis Roque Klering & $\begin{array}{l}\text { - Programa de Pós-Graduação em Administração - } \\
\text { Universidade Federal do Rio Grande do Sul }\end{array}$ \\
\hline Luiz Marcelo Videiro Vieira Santos & - Mestrando em Administração Pública e Governo - EAESP/FGV \\
\hline Marlene Libardoni & - Ações em Gênero, Cidadania e Desenvolvimento - AGENDE \\
\hline Nelson Luiz Nouvel Alessio & - Mestre em Administração Pública e Governo - EAESP/FGV \\
\hline Pedro Jacobi & $\begin{array}{l}\text { - Centro de Estudos e Pesquisas em } \\
\text { Educação, Cultura e Ação Comunitária - CENPEC }\end{array}$ \\
\hline Ricardo Ernesto Vasquez Beltrão & $\begin{array}{l}\text { - Doutorando em Serviço Social } \\
\text { Pontifícia Universidade Católica de São Paulo - PUC-SP }\end{array}$ \\
\hline Roberta Clemente & - Mestranda em Administração Pública e Governo - EAESP/FGV \\
\hline Silvio Caccia Bava & - PÓLIS - Instituto de Estudos, Formação e Assessoria em Políticas Sọciais \\
\hline Thereza Lobo & - Centro de Estudos de Políticas Públicas - CEPP \\
\hline
\end{tabular}




\begin{tabular}{|c|c|}
\hline Ailton Krenak & - Núcleo de Cultura Indígena \\
\hline Carlos Eduardo Evangelisti Mauro & - Mestrando em Administração Pública e Governo - EAESP/FGV \\
\hline Dilsea Adeodata Bonetti & $\begin{array}{l}\text { - Programa de Estudos Pós-Graduados em Serviço Social } \\
\text { Pontíficia Universidade Católica de São Paulo - PUC-SP }\end{array}$ \\
\hline Fernando Guilherme Tenório & $\begin{array}{l}\text { - Escola Brasileira de Administração Pública - } \\
\text { Fundação Getulio Vargas - EBAP/FGV }\end{array}$ \\
\hline Grazia de Grazia & - FASE - Federação de Órgãos para Assistência Social e Educacional \\
\hline Humberto Marques Filho & $\begin{array}{l}\text { - Programa de Pós-Graduação em Administração - } \\
\text { Universidade Federal da Paraíba }\end{array}$ \\
\hline Ilka Camarotti & - Subprograma Práticas Públicas e Pobreza - EAESP/FGV \\
\hline José Antônio Gomes de Pinho & $\begin{array}{l}\text { - Núcleo de Pesquisa e Pós-Graduação em Administração - } \\
\text { Universidade Federal da Bahia }\end{array}$ \\
\hline José Carlos Vaz & - PÓLIS - Instituto de Estudos, Formação e Assessoria em Políticas Sociais \\
\hline Laura da Veiga & - Escola de Governo de Minas Gerais - Fundação João Pinheiro \\
\hline Luis Mario Fujiwara & - Mestrando em Administração Pública e Governo - EAESP/FGV \\
\hline Luis Roque Klering & $\begin{array}{l}\text { - Programa de Pós-Graduação em Administração - } \\
\text { Universidade Federal do Rio Grande do Sul }\end{array}$ \\
\hline Maria Magdalena Alves & - Ação da Cidadania - São Paulo \\
\hline Marlene Libardoni & - Ações em Gênero, Cidadania e Desenvolvimento - AGENDE \\
\hline Nancy Cardia & - Universidade de São Paulo - Núcleo de Estudos da Violência \\
\hline Nelson Luiz Nouvel Alessio & - Mestre em Administração Pública e Governo - EAESP/FGV \\
\hline Pedro Jacobi & $\begin{array}{l}\text { - Centro de Estudos e Pesquisas em } \\
\text { Educação, Cultura e Ação Comunitária - CENPEC }\end{array}$ \\
\hline Ricardo Ernesto Vasquez Beltrão & $\begin{array}{l}\text { - Doutorando em Serviço Social } \\
\text { Pontifícia Universidade Católica de São Paulo - PUC-SP }\end{array}$ \\
\hline Roberta Clemente & - Mestranda em Administração Pública e Governo - EAESP/FGV \\
\hline Rubens Mazon & $\begin{array}{l}\text { - Escola de Administração de Empresas de São Paulo - } \\
\text { Fundação Getulio Vargas - EAESP/FGV }\end{array}$ \\
\hline Silvia Regina Costa Salgado & - CEPAM - Fundação Prefeito Faria Lima \\
\hline
\end{tabular}




\section{VISITAS DE CAMPO}

Adalberto de Oliveira Brandão

Curso de Serviço Social na Universidade Vale do Rio Doce UNIVALE (Governador Valadares / MG)

Alvino Oliveira Sanches Filho Escola de Administraçăo da Universidade Federal da Bahia

Antônio Sérgio Araújo Fernandes

Doutorando em Ciência Política na Universidade de São Paulo - USP

Carla Bronzo Ladeira Carneiro Doutoranda em Sociologia e Política na Universidade Federal de Minas Gerais

Grazia de Grazia

FASE - Federação de Órgãos

para Assistência Social e Educacional

Heitor Paulo Battaglia

Mestre em Administração Pública e Governo - EAESP/FGV

István Van Deursen Varga

Mestrado em Saúde e Ambiente

da Universidade Federal do Maranhão - UFMA

Ivanete Amaral Silva

Mestranda em Planejamento do Desenvolvimento

no Núcleo de Altos Estudos Amazônicos - NAEA, da Universidade Federal do Pará - UFPA

José Raimundo B. Trindade

Núcleo de Altos Estudos Amazônicos - NAEA,

da Universidade Federal do Pará - UFPA

Lorenzo Zanetti

FASE - Federação de Órgãos

para Assistência Social e Educacional

Luis Mario Fujiwara

Mestrando em Administração Pública e Governo - EAESP/FGV

Luiz Marcelo Videiro Vieira Santos

Mestrando em Administração Pública e Governo - EAESP/FGV

Marco Antonio Carvalho Teixeira

Doutorando em Ciências Sociais

na Pontifícia Universidade Católica de São Paulo - PUC-SP

Maria Ceci Misoczky

Departamento de Medicina Social

da Universidade Federal do Rio Grande Sul

Nelson Luiz Nouvel Alessio

Mestre em Administração Pública e Governo - EAESP/FGV

Ricardo Ernesto Vasquez Beltrão

Doutorando em Serviço Social

na Pontifícia Universidade Católica de São Paulo - PUC-SP

Roberta Clemente

Mestranda em Administração Pública e Governo - EAESP/FGV

Vânia Santana

FASE - Federação de Órgãos

para Assistência Social e Educacional

Veronika Paulics

PÓLIS - Instituto de Estudos, Formação

e Assessoria em Políticas Sociais

Zugleimia del Carmen Hidalgo

Mestrando em Administração Pública

na Escola Brasileira de Administração Pública - EBAP/FGV
- Combatendo a Evasão Escolar,

do municipio de Santa Maria / RS

- Plano de Trabalho da Promotoria de Defesa Comunitária. do Estado de Goiás

- Manejo Integrado da Bacia Hidrográfica do Rio Almada, dos municípios de Almadina, Coaraci e Uruçuca / BA

- Projeto Casa de Zabelê,

do municipio de Teresina / PI

- República Bem-Viver,

do município de Santos / SP

- Educação na Prisão.

do Distrito Federal

- Trienal de Medicina Tradicional do Povo Baniwa e Kuripako, das Comunidades Baniwas e Kuriopakos do Rio Içana / AM

- Projeto Parteiras Tradicionais do Amapá, do Estado do Amapá

- Projeto Cidadão.

do Estado do Acre

- Núcleo Intersindical de Conciliação Trabalhista, do município de Patrocínio / MG

- Programa de Fomento a Pequenos Negócios / Setor Informal, do Estado do Espírito Santo

- Recuperação da Agricultura Tradicional e seus Valores Culturais, da União das Aldeias Krahó-Kapey, em Itacajá / TO

- Programa Autogestão da Limpeza Pública,

do município de Timóteo / MG

- Projeto de Saúde Mental, do município de Belo Horizonte / MG

- Desenvolvimento Auto-Sustentável na Pré-Amazônia Maranhense, do Estado de Maranhão

- Convênio Creches Comunitárias, do município de Porto Alegre / RS

- Câmara do Grande ABC,

dos municípios da Região do Grande ABC / SP

- Casa do Caminho - Abrigo para Mulheres Vitimas de Violência, do Estado do Ceará

- Programa Mala do Livro - Bibliotecas Domiciliares, do Distrito Federal

- Reforma Agrária Solidária, do Estado do Ceará 
FINALISTAS - ÓRGÃOS E PESSOAS RESPONSÁVEIS PELAS INSCRIC̣ÕES E PELA APRESENTAÇÃO DOS PROGRAMAS À BANCA JULGADORA

Anamara Simões Martins (Secretária de Ação Comunitária) Gisela lone dos Santos (Assistente Social)

André Fernando (Presidente)

Ângelo Roncalli de Ramos Barros (Diretor Executivo)

Antônio Bezerra Peixoto (Superintendente)

José Lima Castro Júnior (Diretor)

Wilson V. Brandão Junior

Antonio Carlos Guedes (Pesquisador - EMBRAPA)

Fernando Schiavini (Indigenista - FUNAI)

Getulio Orlando Pinto Krahó

Oscar Krahó Rapor (Coordenador da Kapey)

Antônio Gomes de Vasconcelos (Fundação CEMEAR)

Roberto Queiroz do Nascimento (Prefeito Municipal de

Patrocínio)

Arquilau de Castro Melo (Desembargador - Coordenador do Projeto)

Eliezer Mattos Scherrer (Desembargador - Presidente em

Exercício)

Raimundo Angelim Vasconcelos

Benício Marques de Souza (Gerente)

Fernando Augusto Barros Betarello (Diretor-Presidente)

Maria de Lourdes Buaiz

Carla Simone Miranda Borges (Coordenadora)

Iolanda Avelino de Carvalho Sá (Secretária-Adjunta)

Celso Daniel (Prefeito Municipal de Santo André)

Mauricio Soares (Prefeito Municipal de São Bernardo do

Campo - Presidente do Consórciol

César Henrique Santos Pires (Reitor da UEMA)

Hamilton Jesus Santos Almeida (Gerente do Núcleo de

Biotecnologia Agronômica)

Cleide Cristina Soares (Coordenadora)

Hamilton Pereira da Silva (Secretário)

Demóstenes Lázaro Xavier Torres (Procurador-Geral de Justiça do Estado de Goiás)

Rúbian Corrêa Coutinho (Promotora de Justiça)

Elias Carlos Lopes (Diretor Administrativo)

José Martins Filho (Presidente)

Fausto Pereira dos Santos (Secretário Adjunto)

Miriam Nadim Abou-Yd (Coordenadora)

Janete Maria Góes Capiberibe (Primeira-Dama do Estado)

Maria Otacilia L. Battistelli

José Marcos Luedy Oliveira (Gerente Técnico)

José Raimundo Laudano Santos (Prefeito Municipal de

Almadina)

Wallace Coelho Setenta

Judite Guerra (Assessora Pedagógica)

Sofia Cavedon Nunes (Secretária Adjunta)

Luci Beatriz Zelada Duartes (Supervisora Pedagógica)

Pedro Lecueder Aguirre (Secretário Municipal)

Valmir Beltrame (Supervisor Pedagógico)

Maria Jaqueline Maia Pinheiro (Coordenadora)

Tânia de Fátima Gurgel Nobre (Presidente)
- República Bem-Viver

Secretaria de Ação Comunitária, de Santos / SP

- Trienal de Medicina Tradicional do Povo Baniwa e Kuripako Organização Indígena da Bacia do Içana - OIBI, do Amazonas

- Educação na Prisão

Fundação de Amparo ao Trabalhador Preso - FUNAP, do Distrito Federal

- Reforma Agrária Solidária Instituto de Desenvolvimento Agrário do Ceará - IDACE

- Recuperação da Agricultura Tradicional e seus Valores Culturais Uniăo das Aldeias Krahó-Kapey, de Itacajá / TO

- Núcleo Intersindical de Conciliação Trabalhista Fundação Cemear, de Patrocínio / MG

- Projeto Cidadão

Tribunal de Justiça do Estado do Acre

- Programa de Fomento a Pequenos Negócios / Setor Informal Banco de Desenvolvimento do Espírito Santo S/A - BANDES

- Projeto Casa de Zabelê

Secretaria Municipal da Criança e do Adolescente. de Teresina / PI

- Câmara do Grande ABC

Consórcio Intermunicipal do Grande ABC

- Desenvolvimento Auto-Sustentável na Pré-Amazônia Maranhense Universidade Estadual do Maranhăo - UEMA

- Programa Mala do Livro - Bibliotecas Domiciliares Secretaria de Cultura e Esporte do Distrito Federal

- Plano de Trabalho da Promotoria de Defesa Comunitária Ministério Público do Estado de Goiás

- Programa Autogestão da Limpeza Pública Conselho Comunitário do Município de Timóteo

- Projeto de Saúde Mental Secretaria Municipal de Saúde, de Belo Horizonte

- Projeto Parteiras Tradicionais do Amapá Coordenadoria de Articulação com a Sociedade Civil Organizada

- Manejo Integrado da Bacia Hidrográfica do Rio Almada Prefeitura Municipal de Almadina

- Convênio Creches Comunitárias

Secretaria Municipal de Educação - SMED, de Porto Alegre / RS

- Combatendo a Evasão Escolar

Secretaria de Município da Educação de Santa Maria

- Casa do Caminho - Abrigo para Mulheres Vítimas de Violência Fundação da Ação Social - FAS, do Ceará 